Bull. Mater. Sci., Vol. 22, No. 3, May 1999, pp. 495-501. (C) Indian Academy of Sciences.

\title{
Nanocrystalline materials for high temperature soft magnetic applications: A current prospectus
}

\author{
M E McHENRY*, M A WILLARD, H IWANABE ${ }^{\ddagger}, \mathrm{R}$ A SUTTON ${ }^{\ddagger}, \mathrm{Z}$ TURGUT, A HSIAO \\ and D E LAUGHLIN \\ Materials Science and Engineering, Carnegie Mellon University, Pittsburgh, PA 15213-3890, USA \\ ${ }^{\dagger}$ Currently at Japan Electric Corporation, Japan \\ ${ }^{\ddagger}$ Currently at University of Queensland, Australia
}

\begin{abstract}
Conventional physical metallurgy approaches to improve soft ferromagnetic properties involve tailoring chemistry and optimizing microstructure. Alloy design involves consideration of induction and Curie temperatures. Significant in the tailoring of microstructure is the recognition that the coercivity, $\left(H_{\mathrm{c}}\right)$ is roughly inversely proportional to the grain size $\left(D_{\mathrm{q}}\right)$ for grain sizes exceeding $\sim 0.1-1 \mu \mathrm{m}$ (where the grain size exceeds the Bloch wall thickness, $\delta$ ). In such cases grain boundaries act as impediments to domain wall motion, and thus fine-grained materials are usually harder than large-grained materials. Significant recent development in the understanding of magnetic coercivity mechanisms have led to the realization that for very small grain sizes $D_{\mathrm{g}}<\sim 100 \mathrm{~nm}, H_{\mathrm{c}}$ decreases sharply with decreasing grain size. This can be rationalized by the extension of random anisotropy models that were first suggested to explain the magnetic softness of transition-metal-based amorphous alloys. This important concept suggests that nanocrystalline and amorphous alloys have significant potential as soft magnetic materials. In this paper we have discussed routes to produce interesting nanocrystalline magnets. These include plasma (arc) production followed by compaction and primary crystallization of metallic glasses. A new class of nanocrystalline magnetic materials, HITPERM, having high permeabilities at high temperatures have also been discussed.
\end{abstract}

Keywords. Soft magnetic materials; nanocrystals; NANOPERM, HITPERM, high temperature magnetic materials.

\section{Introduction}

Over several decades amorphous and, more recently, nanocrystalline materials have been investigated for applications in magnetic devices requiring magnetically soft materials, such as transformers, jnductive devices, etc. Chemical and structural variations on a nanoscale are important for determining optimal magnetic properties. In this paper we shall discuss recent developments in the synthesis, structural characterization, properties and applications of nanocrystalline (and amorphous) magnets. We have considered processing routes to control chemistry and microstructural morphology on increasingly smaller length scales, and developing experimental techniques which allow more accurate and quantitative probes of structure (including magnetic domain structure) on smaller length scales.

Demands on bulk soft magnetic materials include higher combined induction and permeabilities, and for some applications magnets capable of operating at higher tem-

*Author for correspondence peratures as well as many other nonmagnetic issues such as mechanical properties, corrosion resistance, etc. For achieving such goals, key issues include alloy chemistry, structure, and more importantly the ability to tailor microstructural features. It is clear that the magnets used in soft magnetic applications must be optimized in terms of their intrinsic and extrinsic magnetic properties as well as their morphology. The key intrinsic magnetic properties, the saturation magnetic induction, $B_{\mathrm{s}}$, and Curie temperature, $T_{\mathrm{C}}$, however, are determined solely by alloy composition.

Premiere high induction bulk materials include $\mathrm{Fe}$, $\mathrm{Fe}-\mathrm{Si}$, $\mathrm{Co}$ and $\mathrm{FeCo}$ alloys (for reviews see Chen 1986; Boll 1994). FeCo bulk alloys (Pfeifer and Radeloff 1980; Rajkovic and Buckley 1981) are particularly attractive because of their high inductions, while their permeabilities are smaller than those of amorphous and nanocrystalline alloys and $\mathrm{Si}$ steels. The extrinsic property of interest is the magnetic permeability, $\mu$; the magnetic response function in an applied field which is usually inversely related to the materials coercivity, $H_{c}$. The magnetic permeability, $\mu$, is determined by chemistry, structure and morphology (shape). In particular, alloys with small 
magnetocrystalline anisotropies and magnetostrictive coefficients give rise to particularly soft magnetic materials.

Choice of soft magnetic materials for applications has been guided by recent developments in the field of soft magnetic materials as summarized in figure 1. Amorphous and nanocrystalline magnetic materials, in terms of combined induction and permeabilities are now competitive with $\mathrm{Si}-\mathrm{Fe}$ bulk alloys, and the above-mentioned $\mathrm{Fe}-\mathrm{Co}$ alloys. In figure 1a (adapted from Makino et al 1997),
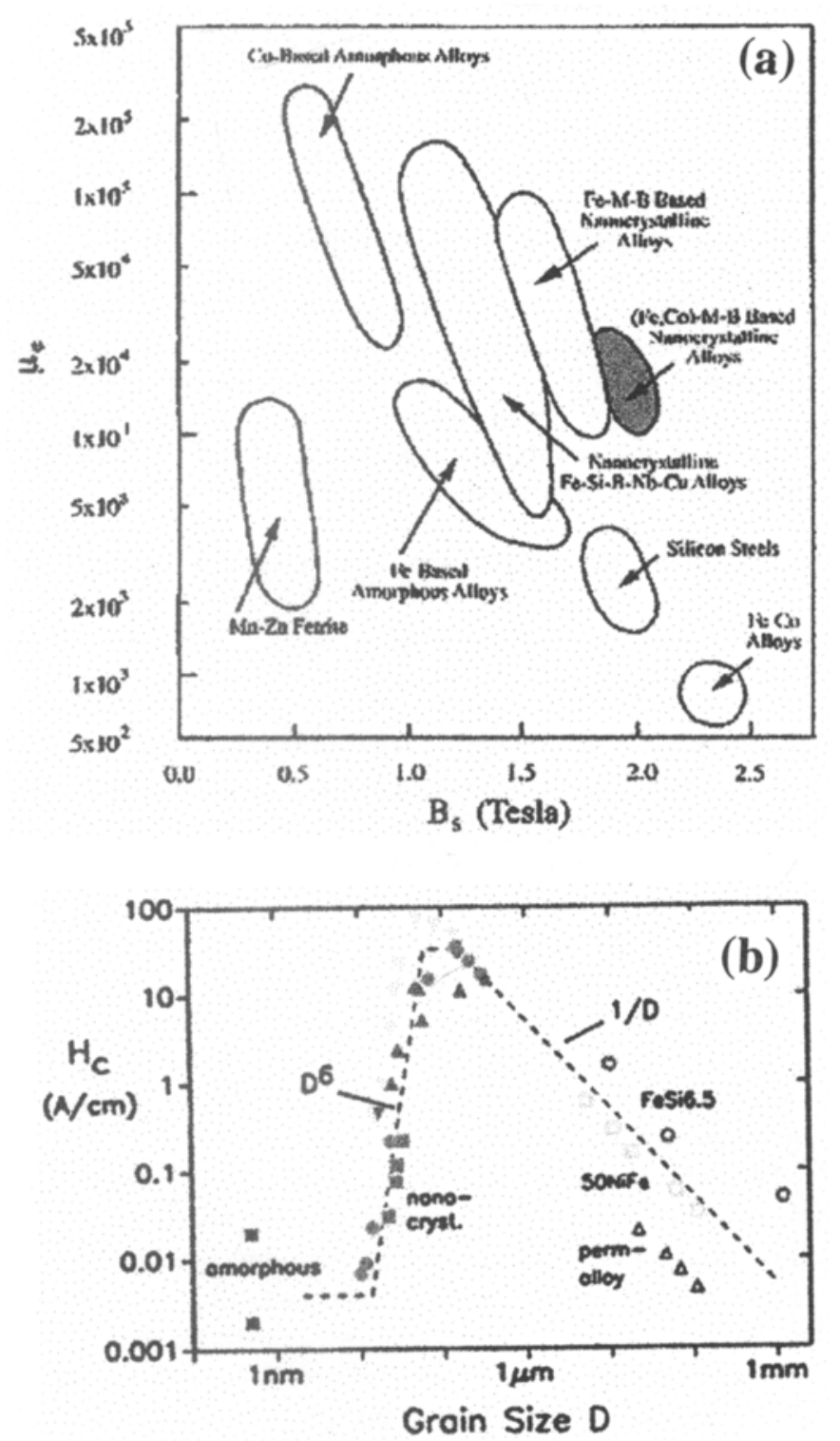

Figure 1. (a) (Adapted from Makino et al 1997). Rclationship between permeability $\mu_{e}$ (at $1 \mathrm{kHz}$ ) and saturation polarization for soft magnetic materials and (b) Herzer diagram illustrating dependence of the coercivity, $H_{\mathrm{c}}$, with grain size in magnetic alloys. figures of merit, $\mathrm{B}_{\mathrm{s}}$ and $\mu_{\mathrm{e}}$, for Co-based amorphous alloys, Fe-based amorphous alloys, and nanocrystalline alloys are summarized, which have evolved over the past decades with soft magnetic properties exceeding those of the bulk alloys based on $\mathrm{Fe}, \mathrm{Co}$, and $\mathrm{Fe}-\mathrm{Co}$. Nanocrystalline $\mathrm{Fe}-\mathrm{Si}-\mathrm{B}-\mathrm{Nb}-\mathrm{Cu}$ alloys have been patented under the trade name FINEMETS@TM (Yoshizawa et al 1988a, b; Yoshizawa and Yamauchi 1989, 1990, 1991). Soft materials, based on FeMBCu, have been patented under the trade name NANOPERM@TM. These $\mathrm{Fe}-\mathrm{M}-\mathrm{B} \quad(\mathrm{M}=\mathrm{Zr}, \mathrm{Nb}, \mathrm{Hf}, \ldots)$ nanocrystalline alloys have all been optimized to achieve small magnetostrictive coefficients with concomitant large permeabilities. More recently ( $\mathrm{Fe}, \mathrm{Co}) \mathrm{MBCu}(\mathrm{M}=\mathrm{Nb}, \mathrm{Hf}$, or $\mathrm{Zr}$ ) nanocrystalline alloys, called HITPERM have been shown to have attractive inductions $(1 \cdot 6-2 \cdot 1 \mathrm{~T})$ combined with high permeabilities and high Curie temperatures. In FINEMETS $\alpha$-FeSi nanoparticles with a $\mathrm{DO}_{3}$ structure are observed, in NANOPERM $\alpha$-Fe particles with a bcc structure are formed. In HITPERM alloys (Willard 1998, 1999 , to appear) nanocrystalline $\alpha^{\prime}$-FeCo are formed with significantly improved high temperature magnetic properties than in the former two.

Conventional physical metallurgy approaches to improve soft ferromagnetic properties involve tailoring chemistry and optimizing microstructure. Significant in the tailoring of microstructure is the recognition that a measure of the magnetic hardness (the coercivity, $H_{\mathrm{c}}$ ) is roughly inversely proportional to the grain size $\left(D_{\mathrm{g}}\right)$ for grain sizes exceeding $\sim 0 \cdot 1-1 \mu \mathrm{m}$ (where the grain size exceeds the Bloch wall thickness, $\delta$ ). In such cases grain boundaries act as impediments to domain wall motion, and thus fine-grained materials are usually harder than large-grained materials.

Significant recent development in the understanding of magnetic coercivity mechanisms have led to the realization that for very small grain sizes $D_{\mathrm{g}}<\sim 100 \mathrm{~nm}$, (Herzer and Hilzinger 1986, 1989; Herzer 1990, 1992, 1995, 1997), $H_{\mathrm{c}}$ decreases quickly with decreasing grain size (figure $1 \mathrm{~b}$ ). This can be rationalized by the extension of random anisotropy models (Alben et al 1978a, b) that were first suggested to explain the magnetic softness of transition-metal-based amorphous alloys. This is illustrated by the fact that the domain wall, whose thickness $\delta$, which exceeds the grain size, now samples several or many grains so that fluctuations in magnetic anisotropy on the crystal length scale are irrelevant to domain wall pinning. This important concept suggests that nanocrystalline and amorphous alloys have significant potential as soft magnetic materials. Soft magnetic properties require that nanocrystalline grains be exchange coupled, and therefore any of the processing routes yielding free-standing nanoparticles must be coupled with a compaction method in which the magnetic nanoparticles end up exchange coupled. 
In this paper we shall explore and illustrate issues that are pertinent to the general understanding of the magnetic properties of nanocrystalline materials. The development of nanocrystalline materials for soft magnetic applications is an emerging field for which we will try to offer a current perspective that is expected to evolve further with time.

\section{Alloy design}

Alloy design includes issues of chemistry and processing designed to optimize one of a number of important intrinsic and/or extrinsic magnetic properties as well/or to optimize structural or microstructural features which promote important (usually extrinsic) magnetic properties. The first of these issues concerns the choice of chemistry so as to have an impact on the intrinsic magnetization of the material. The second issue involves alloy additions designed to aid in the formation of an amorphous or bulk amorphous phase, as a means to an end or as a precursor to producing a nanocrystalline material.

\subsection{Intrinsic magnetic properties}

The desire for large inductions limits choices of alloys to those rich in $\mathrm{Fe}$ or $\mathrm{Co}$ and therefore near the top of the Slater-Pauling curve. As described in the SlaterPauling curve, Fe-Co alloys exhibit the largest magnetic inductions on any material, and also have $T_{c}$ 's desirable for high temperature applications. Alloys near the equiatomic composition are particularly soft and exhibit large permeabilities, but this magnetic softness is rooted in a zero crossing of the first-order magnetic anisotropy constant, $K_{i}$. Fe-rich alloys typically have smaller inductions and lower Curie temperatures than $\mathrm{Fe}-\mathrm{Co}$ alloys. For applications such as rotor assemblies in the more electric aircraft (Quigley) where operation at temperatures up to $600^{\circ} \mathrm{C}$ are desired no other alloy system offers significant potential for these applications. For this reason our group has concentrated on nanocrystalline alloys based on FeCo.

$\mathrm{R}$ A Sutton (unpublished) has used LKKR band structure calculations to determine local moments on $\mathrm{Fe}$ and $\mathrm{Co}$ in the disordered and ordered $\mathrm{B} 2 \mathrm{Fe}_{1-x} \mathrm{Co}_{x}$ alloys. The local moment on Fe was found to be sensitive to composition, while that of $\mathrm{Co}$ is relatively invariant with composition (figure 2a). The net alloy moment reproduces the trends of the famous Slater-Pauling curve. An approximately $4 \%$ larger alloy moment is observed for equiatomic $\mathrm{FeCo}$ in the ordered $\mathrm{B} 2(\mathrm{CsCl})$ structure. Mean field theory fits to temperature-dependent magnetization data for equiatomic FeCo (figure 2b), predicts that equiatomic FeCo would have a Curie temperature of $1475 \mathrm{~K}$ if it could be stabilized with respect to the $\alpha \rightarrow \gamma$ phase transition. This large $T_{v}$ for FeCo leads to a very flat $M(T)$ at temperatures of interest for the MORE electric plane $\left(550-600^{\circ} \mathrm{C}\right)$.
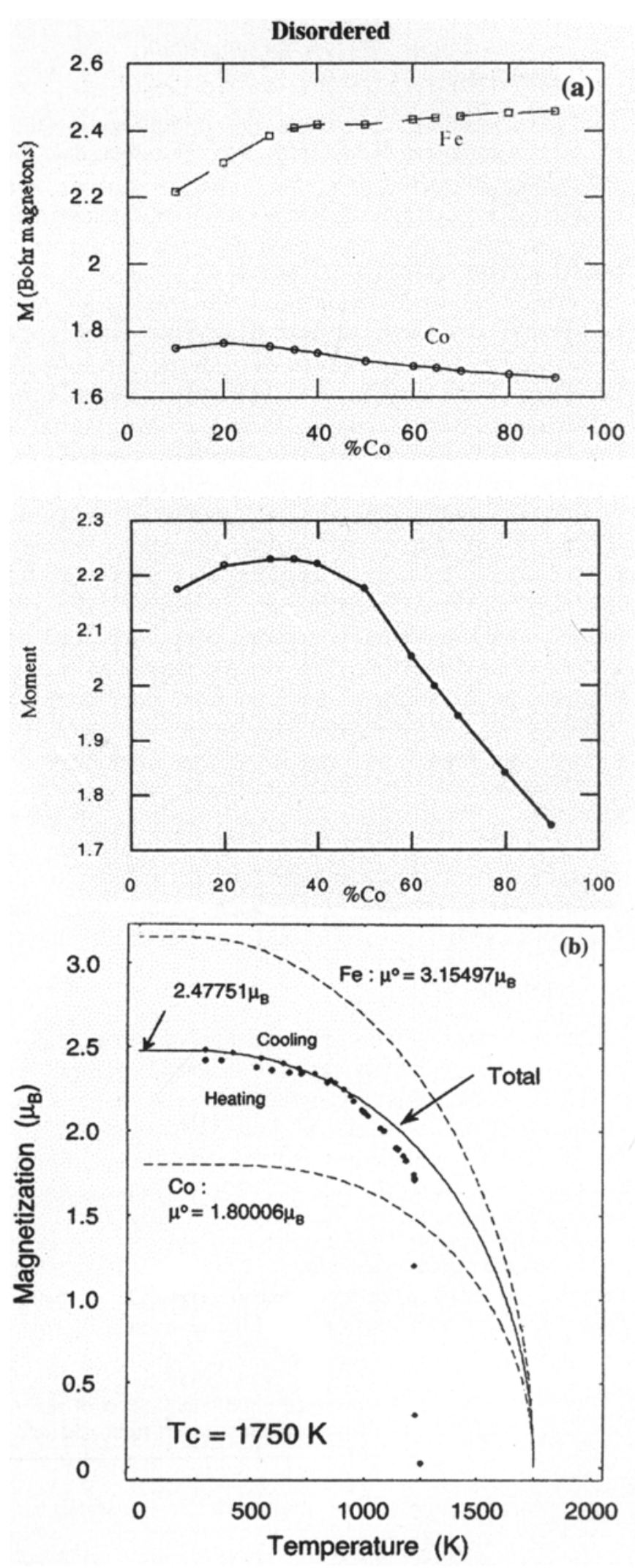

Figure 2. (a) Spin-only Slater-Pauling curve for a disordered $\mathrm{Fe}-\mathrm{Co}$ alloy as determined from LKKR band structure calculations ( $R$ A Sutton, unpublished data), Co-site, Fe-site and total moment as a function of composition and (b) mean field theory fit to temperature dependent magnetization data for equiatomic $\mathrm{FeCo}$ using moments of (a). 


\subsection{Considerations of glass forming ability and} crystallization

The considerations for synthesizing amorphous metallic alloys are often at odds with the considerations for optimizing magnetic properties. This is because the addition of typical glass forming agents has a deleterious effect on such intrinsic magnetic properties as the saturation induction and the Curie temperature. Considerations for glass forming ability in binary systems include the temperature and composition of the eutectic, the terminal phases to the right and left of the eutectic composition, the slope of the liquidus as a function of composition, etc. If the amorphous alloy is to be used as a precursor for the production of a nanocrystalline material, the primary and secondary crystallization temperatures are of importance as is the structure of the ferromagnetic nanocrystalline phase that is the product of primary crystallization. Table 1 summarizes these parameters for selection of binary Fe-early transition metal (TE) and metalloid (M) systems. Significant alloy systems include $\mathrm{Fe}(\mathrm{Co})-\mathrm{TE}-\mathrm{B}-\mathrm{Cu}$ alloys wherein early transition metals (TE) and $\mathrm{B}$ are added as glass formers, and where $\mathrm{Cu}$ is added to provide nanocrystal nucleation sites. The low TE eutectic concentration in $\mathrm{Fe}(\mathrm{Co})-\mathrm{TE}$ systems is fortuitous in that it implies relatively small moment reduction in these alloys. In crystallization of these alloys, $a-\mathrm{Fe}$ or $a^{\prime}-\mathrm{FeCo}$ nanocrystals are thought to nucleate on $\mathrm{Cu}$ clusters expelling $\mathrm{Zr}$ and $\mathrm{B}$ to an intergranular amorphous phase. Such systems form the basis for FINEMET, NANOPERM, and HITPERM alloys.

\section{Synthesis}

Common synthesis routes for producing amorphous and/or nanocrystalline materials for soft magnetic applications which are viable for producing bulk alloys for larger-scale applications, as opposed to thin film synthesis for microelectronic applications, include the following routes (though others exist) for example. These synthesis routes are:

(I) Powder synthesis techniques: Magnetic nanocrystals may be synthesized as free-standing powders or nanoencapsulates, which must then be compacted to form a bulk alloy with nanocrystalline grains. Selected powder synthesis techniques that include $\mathrm{C}$-arc synthesis (McHenry et al 1994, 1996, 1998), plasma torch synthesis, and mechanical milling techniques.

(II) Rapid solidification processing: Amorphous alloys can be produced by a variety of rapid solidification processing routes. These typically require cooling rates of $>10^{4} \mathrm{~K} / \mathrm{s}$ for typical alloys at a eutectic composition. Slower cooling rates are possible for bulk amorphous alloys.

(III) Solidification processing of bulk amorphous alloys: Bulk amorphous alloys are formed by more conventional solidification routes with slower cooling rates. The socalled large glass forming abilities of these alloys allow for production of amorphous materials with much larger dimensions, thus the name bulk amorphous alloys.

(IV) Crystallization of amorphous precursors: Nanocrystalline bulk alloys with nanocrystalline grains can also be produced by solid state reaction (crystallization) of an amorphous precursor. Our group at CMU has concentrated on routes (I), (II) and (IV) for the synthesis of nanocrystalline magnets. Plasma torch synthesis produces free-standing nanoparticles which must be followed by compaction to produce bulk magnets. Melt spinning produces an amorphous precursor which is then nanocrystallized to yield a two-phase nanocrystalline/ amorphous magnetic material.

\section{Structure and magnetic properties}

Gallagher et al (1996) have produced $\mathrm{Fe}_{x} \mathrm{Co}_{1-x}[\mathrm{C}]$ ( $x=0.0,0.2,0.4,0.5,0.6$, and 0.8 , nominally) freestanding nanoparticles by a Kratschmer-Huffman carbon arc method. X-ray diffraction indicated a single-disordered bcc FeCo phase (along with graphitic C), for all but the $x=0.2$ composition for which some fcc Co was observed. Particles had an average diameter of $50 \mathrm{~nm}$ from Scherrer analysis of the peak widths, and a broad size distribution based on TEM. Imaging energy-filtered microscopy showed $\mathrm{Fe}$ and $\mathrm{Co}$ to be uniformly distributed in the nanoparticles. A compositional dependence of the $M(H=1 \mathrm{~T})$ (figure $3 \mathrm{~b})$ magnetization was similar to that observed in the bulk alloy Slater-Pauling curve. $\mathrm{Fe}_{0.5} \mathrm{Co}_{0.5}[\mathrm{C}]$ exhibited the largest magnetization heretofore

Table 1. Considerations for select glass forming ability in binary systems: temperature and composition of the eutectic, the slope of the liquidus, and the terminal phases to the right and left of the eutectic composition.

\begin{tabular}{lccccl}
\hline $\begin{array}{l}\text { Binary } \\
\text { alloy }\end{array}$ & $\begin{array}{c}x_{\mathrm{E}} \text { at\% } \\
(\mathrm{wt} \%)\end{array}$ & $T_{\mathrm{E}}(\mathrm{C})$ & $\mathrm{d} T_{\mathrm{L}} / \mathrm{d} x$ & $\begin{array}{c}\text { Terminal } \\
\text { phases }\end{array}$ \\
\hline $\mathrm{Fe}-\mathrm{B}$ & $17(3.8)$ & 1174 & 21.4 & $\mathrm{Fe}$ & $\mathrm{Fe}_{2} \mathrm{~B}$ \\
$\mathrm{Fe}-\mathrm{Si}$ & $33(20)$ & 1212 & $\sim 10$ & $\mathrm{Fe}$ & $\mathrm{Fe}_{2} \mathrm{Si}_{2}\left(\mathrm{Fe}_{3} \mathrm{Si}\right)$ \\
$\mathrm{Fe}-\mathrm{Zr}$ & $10(15)$ & 1337 & 20 & $\mathrm{Fe}$ & $\mathrm{Fe}_{2} \mathrm{Zr}\left(\mathrm{Fe}_{3} \mathrm{Zr}\right)$ \\
$\mathrm{Fe}-\mathrm{Hf}$ & $8.5(22)$ & 1390 & 18 & $\mathrm{Co}$ & $\lambda$ \\
$\mathrm{Fe}-\mathrm{Nb}$ & $12(18.5)$ & 1373 & $\sim 14$ & $\mathrm{Fe}$ & $\varepsilon$ \\
Fe-Ta & $7.9(21)$ & 1442 & 12.5 & $\mathrm{Fe}$ & $\varepsilon$ \\
\hline
\end{tabular}


observed in similarly produced nanoparticles. The $M(H)$ curves did not saturate, presumably due to some fraction of superparamagnetic particles, or from canted interfacial spins. These FeCo containing soots, reported by Gallagher et al (1996), had over $200 \mathrm{emu} / \mathrm{g}$ magnetizations (note that $\alpha-\mathrm{Fe}$ has a $\sim 220 \mathrm{emu} / \mathrm{g}$ saturation magnetization) (figure $3 \mathrm{a}$ ).
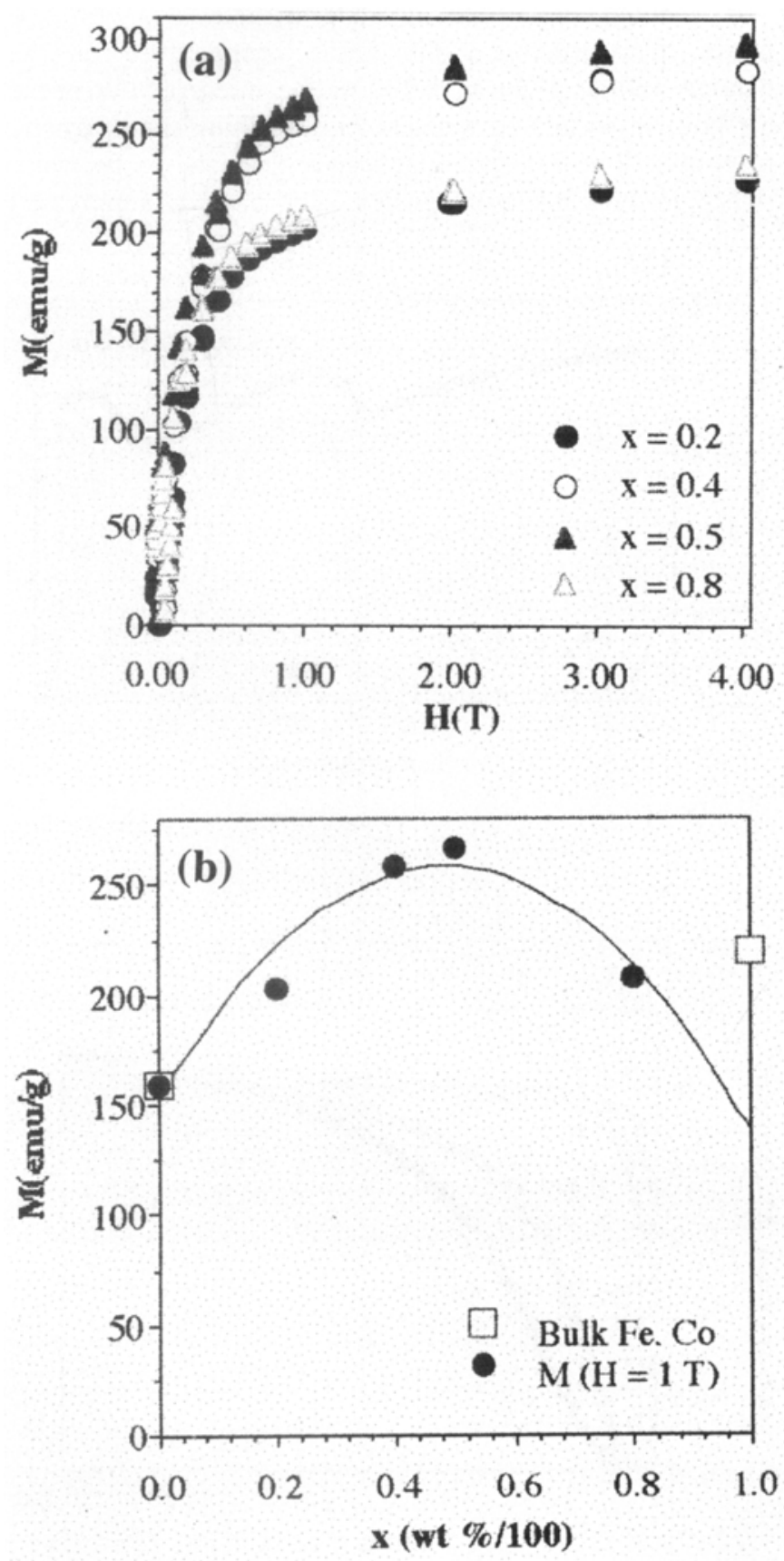

Figure 3. (a) $5 \mathrm{~K} \quad M(H)$ of $\mathrm{Fe}_{x} \mathrm{Co}_{1-x}[\mathrm{C}]$ nanoparticles $(x=0.2,0.4,0.5,0.6$, wt $\% / 100)$ and $(b) M(H=1 \mathrm{~T})$ vs $x$ for nanoparticles including data for bulk $\mathrm{Fe}$ and $\mathrm{Co}$ for comparison. Parabola is a guide to the eye (reproduced from Gallagher et al 1996).
$\mathrm{Fe}-\mathrm{Co}$ alloys undergo an order-disorder phase transformation at a maximum temperature of $725^{\circ} \mathrm{C}$ at the composition $\mathrm{Fe}_{50} \mathrm{Co}_{50}$, with a change in structure from the disordered $\alpha$-bcc (A1) to the ordered $\alpha^{\prime}$-CsCl(B2)-type structure (figure 4a). C-coated $\mathrm{Fe}_{x} \mathrm{Co}_{1-x}(x=0.50,0.45$, $0.40,0.35,0.30,0.25$ ) nanoparticles have been produced using a RF plasma torch by Turgut $e$ t al $(1997,1998)$. The only $\mathrm{C}$ source was acetylene used as a carrier gas. Structural determination by X-ray diffraction indicated a single disordered bcc $\alpha$-FeCo phase along with graphitic C for all compositions. EDX analysis (Scott et al 1997) indicated compositional fluctuations of a few atomic \% for individual particles of one nominal composition, which is attributed to starting with elemental rather than alloy precursors. Magnetic hysteresis loops have been measured to $T>1050 \mathrm{~K}$ and revealed relatively high room temperature coercivities (200-400 Oe), with a strong compositional variation similar to that observed in bulk alloys. Larger coercivities are consistent with particles near the monodomain size for these alloys. The $T$ dependence of the magnetization revealed the effects on atomic ordering. Figure $4 \mathrm{~b}$ illustrates $M(T)$, at $H=500 \mathrm{Oe}$, for equiatomic $\mathrm{FeCo}$ nanocrystalline powders measured on a second heating cycle after initial heating to $920^{\circ} \mathrm{C}$. The influence of ordering is observed prominently in features that are quite similar to thermomagnetic observations for bulk $\mathrm{Fe}_{49} \mathrm{Co}_{49} \mathrm{~V}_{2}$ alloys. These features include a discontinuity in $M(T)$ at $\sim 600^{\circ} \mathrm{C}$ (due to chemical ordering) and a return to the extrapolated low temperature branch of the curve at the disordering temperature of $\sim 730^{\circ} \mathrm{C}$.

In general, the mean field theory describing Curie temperatures for nanoparticles is not strongly deviate from that of the bulk materials. However, this may not be true for very small nanoparticles $(<5 \mathrm{~nm})$ where the fraction of surface atomic sites is large, and therefore the reduced coordination of surface atoms should in fact alter the Curie temperature. For most observations in the literature, however, the $T_{\mathrm{c}}$ does not deviate strongly from that of the bulk materials. Of note in figure $4 \mathrm{~b}$ is that at $\sim 950^{\circ} \mathrm{C}$ we observe an abrupt drop in the magnetization that corresponds to the $\alpha \rightarrow \gamma$ structural phase transformation. It can be concluded from the abruptness in the drop of $M(T)$ that the $\mathrm{Fe}_{0.5} \mathrm{Co}_{0.5}$ alloy has a Curie temperature that exceeds the $\alpha \rightarrow \gamma$ phase transformation temperature. This is corroborated by the differential thermal analysis data, where the orderdisorder and $\alpha \rightarrow \gamma$ phase transformations have also been clearly observed.

NANOPERM and HITPERM alloys have been synthesized by Willard et al (1998, 1999 to appear) through nanocrystallization of amorphous precursors. Figure 5a shows observations of magnetization as a function of temperature (Willard et al 1998) for two alloys, one of a NANOPERM composition and the other of a HITPERM 
composition. Initially, the two samples are amorphous, and as the samples are heated the crystallization of the amorphous phase becomes apparent in each alloy. The NANOPERM material has its Curie temperature just above room temperature. The magnetic phase transition is followed by primary crystallization at $T_{x 1} \sim 500^{\circ} \mathrm{C}$, which in turn is followed by secondary crystallization, and then finally the magnetic phase transition for the $\alpha$-Fe nanocrystalline phase at $\sim 770^{\circ} \mathrm{C}$. Thus re-enterant ferromagnetism is observed in amorphous alloys with primary crystallization temperatures above the Curie temperature of the amorphous phase.

Crystallization kinetics of NANOPERM have been studied by Hsiao et al (1998, unpublished) who examined
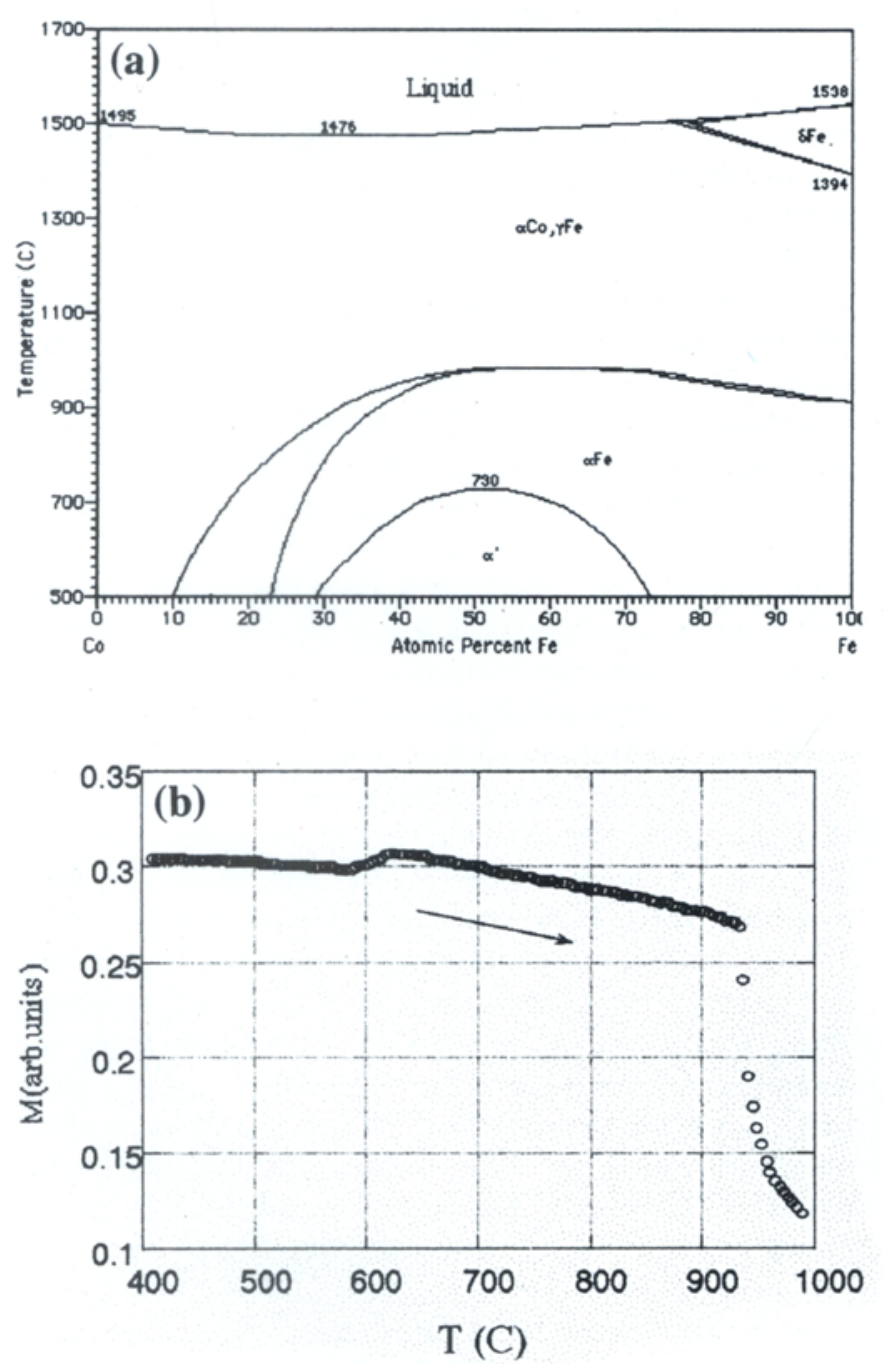

Figure 4. (a) Fe-Co phase diagram (produced using TAPP@TM software, ES Microware) and (b) $M(T)$ data for $\mathrm{C}$-arc synthesized equiatomic $\mathrm{Fe}-\mathrm{Co}$ nanoparticles measured using a Lakeshore model 7300 VSM and oven assembly at $500 \mathrm{Oe}$ measured on a second heating cycle to $995^{\circ} \mathrm{C}$ after initial heating to $920^{\circ} \mathrm{C}$ (Turgut et al 1997). isothermal time-dependent magnetization at temperatures above the crystallization temperature (figure $5 b$ ). Since the amorphous phase is paramagnetic at the crystallization temperature, the magnetization is a direct measure of the volume fraction of the $\alpha$-Fe crystalline phase that has transformed. $M(T)$ then measures the crystallization kinetics. Figure 5 b shows curves reminiscent of JohnsonMehl-Avrami kinetics for phase transformation.

It is noted that in nanocrystalline materials, with two phase microstructures, the Curie temperature of the nanocrystalline phase and that of the intergranular phase are both important parameters in describing the magnetic response. The intergranular phase, which is typically richer in nonmagnetic species, can have a suppressed

(a)

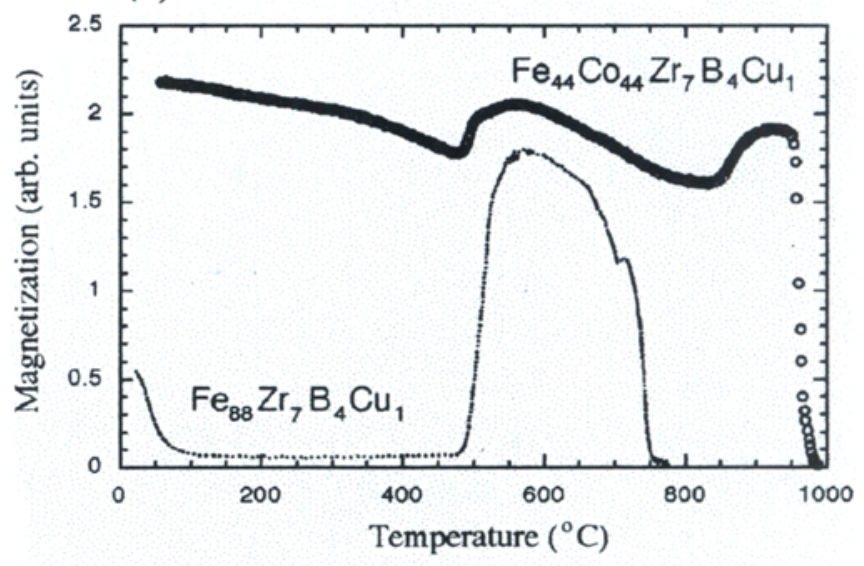

(b)

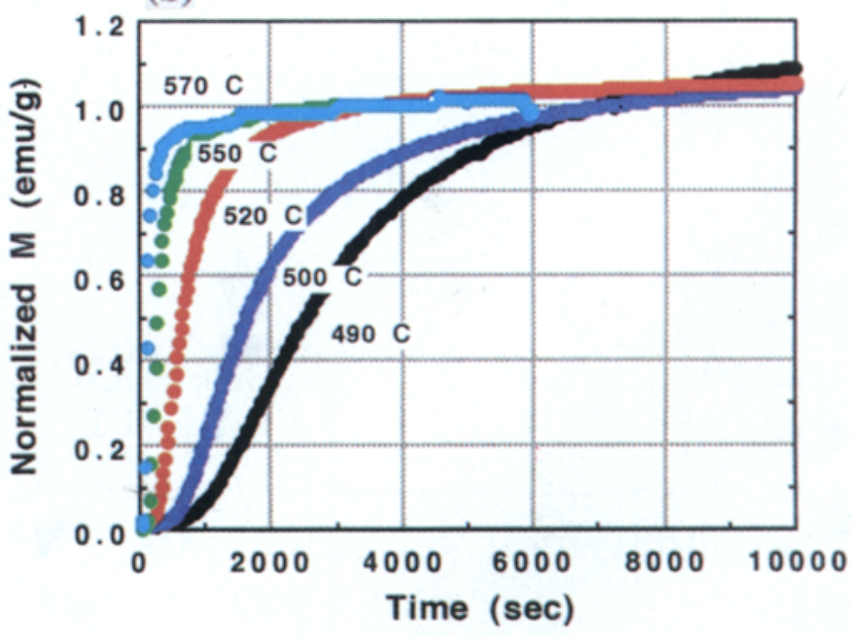

Figure 5. (a) $M(T)$ (Willard et al 1998) for an alloy with a NANOPERM composition $\mathrm{Fe}_{88} \mathrm{Zr}_{7} \mathrm{~B}_{4} \mathrm{Cu}$ and an alloy with a HITPERM composition, $\mathrm{Fe}_{44} \mathrm{Co}_{44} \mathrm{Zr}_{7} \mathrm{~B}_{4} \mathrm{Cu}$, and (b) isothermal magnetization as a function of time for the NANOPERM composition $\mathrm{Fe}_{88} \mathrm{Zr}_{7} \mathrm{~B}_{4} \mathrm{Cu}$ at $490,500,520,550$ and $570^{\circ} \mathrm{C}$, respectively (Hsiao, unpublished data). 
magnetic order parameter and lower Curie temperature than the nanocrystals. This is especially important in describing the temperature dependence of a variety of extrinsic magnetic properties. In figure $5 \mathrm{a}$ the $M(T)$ curve for HITPERM, shows that the magnetization decreases monotonically until $\sim 400^{\circ} \mathrm{C}$ as the amorphous phase approaches its Curie temperature. Above 400$500^{\circ} \mathrm{C}$ structural relaxation and crystallization of the $\alpha^{\prime}$-FeCo phase occurs resulting in larger magnetization due to the larger Curie temperature of the $\alpha^{\prime}$-FeCo phase. The crystallization temperature is apparently well below the Curie temperature of the amorphous phase, so that the magnetization of the amorphous phase is only partially suppressed prior to crystallization. At a temperature corresponding to the $\alpha-\gamma$ phase transition $\left(980^{\circ} \mathrm{C}\right)$, the material abruptly loses its magnetization, consistent with the paramagnetic response of the $\gamma$ phase.

Permeability measurements were made on a HITPERM sample that was prepared as a laminate in a toroidal geometry, and annealed at $600^{\circ} \mathrm{C}$. The frequency dependence of the real and imaginary components of the complex permeability, $\mu^{\prime}$ and $\mu^{\prime \prime}$, reflect the power loss due to eddy currents and hysteretic response. The maximum permeability for this material was determined to be about 1800. $\mu^{\prime \prime}(T)$ peaks at a frequency of $\sim 20 \mathrm{kHz}$. The high peak frequency is thought to reflect the higher resistivity in the nanocrystalline materials compared with conventional bulk alloys. The resistivity is a significant term in eddy current-related damping of domain wall motion.

\section{Conclusions}

$\mathrm{FeCo}$ alloys possess large inductions and Curie temperatures, suggesting their use in high-temperature softmagnetic applications. Free-standing $\mathrm{FeCo}$ nanoparticles with large inductions and flat $M(T)$ to $\sim 970^{\circ} \mathrm{C}$ have been produced by $\mathrm{C}$-arc and plasma torch routes. A new nanocrystalline soft magnetic material, produced by nanocrystallization of an amorphous precursor, with the $\alpha^{\prime}$-FeCo phase (B2 structure) are described with excellent intrinsic magnetic properties and strong intergranular coupling to $600^{\circ} \mathrm{C}$. These have excellent soft magnetic properties to high frequencies and temperatures due to the nanocrystalline grains. This material is therefore a candidate for high temperature aircraft power applications.

\section{Acknowledgements}

MEM thanks the National Science Foundation for support through Grant No. ECD-8907068. Efforts have also been sponsored by the Air Force Office of Scientific Research, Air Force Materiel Command, USAF, under grant number F49620-96-1-0454. The authors acknowledge useful discussions with Prof. S A Majetich, Dr J H Scott, Dr V
Harris, Dr B Lu, Ms K Gallagher, Dr S-Y Chu, and Dr M-Q Huang.

\section{References}

Alben R, Budnick J and Cargill III G S 1978a in Metallic glasses (Ohio: ASM) p. 304

Alben R, Becker J J and Chi M 1978b J. Appl. Phys. 491653

Boll R 1994 Soft magnetic metals and alloys, in Materials science and technology: A comprehensive treatment (ed.) $\mathrm{K} \mathrm{H} \mathrm{J}$ Buschow (Weinhem: VCH) Ch. 14, Vol. 3B, p. 399

Chen C W 1986 Magnetism and metallurgy of soft magnetic materials (New York: Dover Publications Inc.)

Gallagher K, Johnson F, Kirkpatrick E, Scott J H, Majetich S and McHenry M E 1996 IEEE Trans. Mag. 324842

Herzer G 1990 IEEE Trans. Magn. 261397

Herzer G 1991 Mater. Sci. \& Eng. A133 1

Herzer G 1992 J. Magn. Magn. Mater. 112258

Herzer G 1995 IEEE Trans. Mag. 261397

Herzer G 1997 Handbook of magnetic materials (ed.) K H J Buschow (Amsterdam: Elsevier Science) Ch. 3, Vol. 10, p. 415

Herzer G and Hilzinger H R 1986 J. Magn. Magn. Mater. 62 143

Herzer G and Hilzinger H R 1989 Phys. Scr. 39639

Makino A, Hatanai T, Naitoh Y, Bitoh T, Inoue A and Masumoto T 1997 IEEE Trans. Magn. 333793

McHenry M E and Subramoney S 1998 Fullerenes: Physics and technology (eds) K M Kadish and R S Ruoff (New York: John Wiley) (to appear in 1999)

McHenry M E, Majetich S A, De Graef M, Artman J O and Staley S W 1994 Phys. Rev. B49 11358

McHenry M E, Gallagher K, Johnson F, Scott J H and Majetich S A 1996 in Recent advances in the chemistry and physics of fullerenes and related materials, ECS symp. proceedings, PV 96-10, Pennington, $N J$ (eds) K M Kadish and R S Ruoff (N.J.: Pennington) p. 703

Pfeifer F and Radeloff C 1980 J. Magn. Magn. Mater. 19190 Rajkovic M and Buckley R A 1981 Metal Sci. 21

Scott J H, Majetich S A, Turgut Z, McHenry M E and Boulos M 1997 Carbon coated nanoparticle composites synthesized in an RF plasma torch, in Nanostructured materials, MRS proc. (ed.) J C Parker (Pittsburgh, PA: Materials Research Society) (in press)

Turgut Z, Huang M -Q, Gal lagher K, Majetich S A and McHenry M E 1997 J. Appl. Phys. 814039

Turgut Z, Scott J H, Huang M-Q, Majetich S A and McHenry M E 1998 J. Appl. Phys. 836468

Willard M A, Laughlin D E, McHenry M E, Thoma D and Sickafus K 1998 J. Appl. Phys. (to appear)

Willard M A, Laughlin D E and McHenry M E $1999 \mathrm{~J}$. Appl. Phys. (to appear)

Yoshizawa $Y$ and Yamauchi K 1989 J. Jap. Inst. Metals 53241

Yoshizawa Y and Yamauchi K 1990 Mater. Trans. JIM 31307

Yoshizawa Y and Yamauchi K 1991 Mater. Sci. \& Eng. A133 176

Yoshizawa Y, Oguma S and Yamauchi K 1988a J. Appl. Phys. 646044

Yoshizawa Y, Yamauchi K, Yamane T and Sugihara H 1988b J. Appl. Phys. 646047 